\title{
16. Comparison of colloid preloading and continuous infusion of norepinephrine versus crystalloid co-loading and continuous infusion of norepinephrine in the prevention of maternal hypotension
}

Kassiani Theodoraki ${ }^{1, *}$, Sofia Hadzilia ${ }^{2}$, Dimitrios Valsamidis ${ }^{2}$, Konstantina Kalopita $^{2}$, Emmanouil Stamatakis ${ }^{2}$

${ }^{1}$ A' Anaesthesiology Clinic, Pain Relief and Palliative Care Centre, Aretaieion University Hospital, National and Kapodistrian University of Athens, Athens, Greece; ${ }^{2}$ Alexandra General Hospital of Athens, Athens, Greece. * ktheodoraki@hotmail.com

Background and goal of study: Spinal anesthesia for cesarean section can be frequently complicated by hypotension, with untoward effects for both the mother and fetus. Recently, norepinephrine has been shown to be effective in maintaining blood pressure in obstetric patients. Another technique widely used to prevent hypotension is fluid administration. Current evidence suggests that the combination of fluid administration and vasoconstrictive medications should be the main strategy for prevention and management of hypotension. The aim of this randomized study was to investigate the combination of a norepinephrine infusion and colloid preloading versus the combination of a norepinephrine infusion and crystalloid co-loading for the prevention of maternal hypotension during elective cesarean section under combined spinal-epidural anaesthesia

Materials and methods: One hundred parturients were randomized to receive either 6\% hydroxyethyl starch 130/0.4 5 $\mathrm{mL} / \mathrm{kg}$ before spinal anesthesia (colloid preload) or Ringer's Lactate solution $10 \mathrm{~mL} / \mathrm{kg}$ starting with intrathecal injection (crystalloid co-load). Both groups were also administered norepinephrine $4 \mu \mathrm{g} / \mathrm{min}$, starting simultaneously with the administration of the subarachnoid solution. The primary outcome was the incidence of maternal hypotension ( $\mathrm{SBP}<80 \%$ of baseline). The incidence of severe hypotension (SAP $<80 \mathrm{mmHg}$ ), total dose of ephedrine administered as well as maternal side-effects and the acid-base status and Apgar score of the neonate were also recorded

Results: There were no significant differences in the incidence of hypotension $(13.7 \%$ vs. $16.3 \%, P=0.933$ or severe hypotension ( $0 \%$ vs. $4 \%, P=0.238)$ between colloid preload and crystalloid co-load groups, respectively. The median [range] ephedrine dose was 0 [0-15] $\mathrm{mg}$ in the colloid preload group and 0 [0-10] $\mathrm{mg}$ in the crystalloid co-load group $(P$ $=0.807)$. There were no significant differences in maternal side-effects or neonatal outcomes between groups

Conclusion: The incidence of hypotension during elective cesarean section is low and comparable when a norepinephrine infusion is used in combination with either colloid preload or crystalloid co-load, with perhaps a marginal superiority of colloid preload in the prevention of severe hypotension. It appears that the optimal regimen for prevention of maternal hypotension is a combination of fluids and a prophylactic vasopressor like norepinephrine.

\section{References:}

[1] Fitzgerald JP, Fedoruk KA, Jadin SM, Carvalho B, Halpern SH. Prevention of hypotension after spinal anaesthesia for caesarean section: a systematic review and network meta-analysis of randomised controlled trials. Anaesthesia. 2020; 75: 109-121.

[2] Rijs K, Mercier FJ, Lucas DN, Rossaint R, Klimek M, Heesen M. Fluid loading therapy to prevent spinal hypotension in women undergoing elective caesarean section. European Journal of Anaesthesiology. 2020; 37: 1126-1142. 